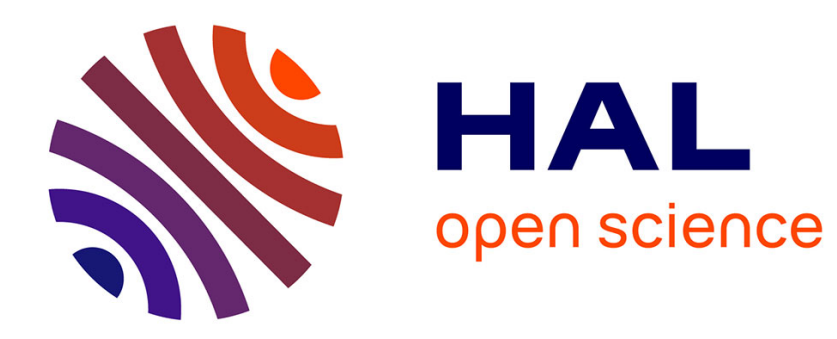

\title{
Errata: Effet d'un champ électrique dans l'hélium 3 superfluide
}

\author{
J.M. Delrieu
}

\section{To cite this version:}

J.M. Delrieu. Errata: Effet d'un champ électrique dans l'hélium 3 superfluide. Journal de Physique Lettres, 1975, 36 (1), pp.22-22. 10.1051/jphyslet:0197500360102201 . jpa-00231143

\section{HAL Id: jpa-00231143 https://hal.science/jpa-00231143}

Submitted on 1 Jan 1975

HAL is a multi-disciplinary open access archive for the deposit and dissemination of scientific research documents, whether they are published or not. The documents may come from teaching and research institutions in France or abroad, or from public or private research centers.
L'archive ouverte pluridisciplinaire HAL, est destinée au dépôt et à la diffusion de documents scientifiques de niveau recherche, publiés ou non, émanant des établissements d'enseignement et de recherche français ou étrangers, des laboratoires publics ou privés. 


\section{ERRATA}

J. Physique Lett. 35 (1974) L-157

«DIFFERENT TYPES OF SMECTIC H PHASES » by A. DE VRIES

Remplacer référence [1] par :

[1] De Vries, A., J. Chem. Phys. 61 (1974) 2367.

J. Physique Lett. 35 (1974) L-189

\section{EFFET D'UN CHAMP ÉLECTRIQUE DANS L'HÉLIUM 3 SUPERFLUIDE}

\section{J. M. DELRIEU}

Une erreur de signe dans la transformée de Fourier de $W_{\mathbf{D}}$ entraîne qu'à partir de la seconde formule $W_{\mathbf{D}}$ doit être changé de signe (sauf dans la formule $\delta W_{\mathrm{D}}=\frac{4 \pi}{10} \alpha^{2}(\mathbf{E} . \mathbf{L})^{2} \int_{0}^{\infty}|C(|\mathbf{k}|)|^{2} \mathrm{dk}$ ). Ceci impose donc que $\left(A^{+} A\right)_{z z}$ soit maximum dans la direction du champ. Le champ électrique oriente donc la phase superfluide dans la direction où le paramètre d'ordre est maximum. Pour la phase $\mathbf{A}$ l'axe orbital $\mathbf{L}$ sera perpendiculaire au champ électrique $\mathbf{E}$ de sorte qu'un champ électrique et magnétique croisés sont nécessaires à la fois pour réaliser un monodomaine où $\mathbf{L}$ est perpendiculaire à ces 2 champs (loin des parois). Dans la phase $\mathbf{B}$ isotrope le principe de la discussion reste valable à part que l'axe de rotation $\mathbf{n}$ de la matrice $A$ est perpendiculaire au champ électrique $\mathbf{E}$ avec une très faible énergie d'anisotropie. Nous tenons à remercier le professeur Legett pour nous avoir fait remarquer cette erreur. 